\title{
On the Space Reuse Efficiency of TSI-free OTDM Rings
}

\author{
An Chen, Albert Kai-Sun Wong, and Chin-Tau Lea
}

\begin{abstract}
Design of future OTDM (Optical Time-Division Multiplexing) rings may require the network nodes to perform the time-slot interchange (TSI) function of moving data from an incoming time-slot to different outgoing time-slots. An efficient optical buffer technology for optical TSI is not currently available. Our previous work [1] has shown that without TSIs, all slots in an OTDM rings will form equal-length cycles. In this letter, we derive a general formula that shows the relationship between the cycle length and the space reuse efficiency of a TSI-free OTDM bi-directional ring.
\end{abstract}

Index Terms-Optical TDM, optical ring architecture.

\section{INTRODUCTION}

$\mathbf{T}$ HE bandwidth granularity offered by WDM (Wavelength Division Multiplexing) systems is coarser than needed in many scenarios. OTDM (optical time-division multiplexing) networks [2][3][4] subdivide the bandwidth of an individual wavelength into time slots within periodic frames. A lightpath in an OTDM network is analogous to a circuit in a traditional TDM system - it occupies and is identified a sequence of allocated time slots as it traverses link to link from source to destination and provides a temporal fraction of the link bandwidth. OTDM requires minimum header processing and is a more practical form of time-domain optical switching in the near future than packet switching [5][6], the latter being hindered by the lack of adequate optical processing devices.

Ring is a commonly used topology for TDM systems. Each node can perform the add/drop operation of inserting and removing data from a slot in the ring. A node often needs to switch the data from an incoming slot to a different outgoing slot. This is the time-slot interchange (TSI) function. An optical TSI is much harder to implement than its electronic counterpart. One implementation is shown in Fig. 1 where the optical TSI consists of an optical switch and fiber-delay-lines. A data block will pass through the switch a variable number of times depending on how long it should be delayed, and suffer from a variable signal attenuation. The size of the buffer grows with the OTDM frame size, which is usually large. The complexity and the cost of the TSI will make an OTDM ring impractical to build at the current time.

If we eliminate the TSI, an OTDM ring needs only simple delay lines at each node to synchronize the incoming and outgoing slots (Fig. 2 ), significantly reducing complexity and cost. A fundamental result we discovered in [1] is that in every

Manuscript received November 5, 2007. The associate editor coordinating the review of this letter and approving it for publication was C. Assi.

C. T. Lea and A. Wong are with the Department of Electronic and Computer Engineering, Hong Kong University of Science and Technology (e-mail: \{eelea, eealbert\}@ece.ust.hk).

A. Chen is with Giant Innovation (HK) Limited (e-mail: anchen@ece.ust.hk).

Digital Object Identifier 10.1109/LCOMM.2008.071842.

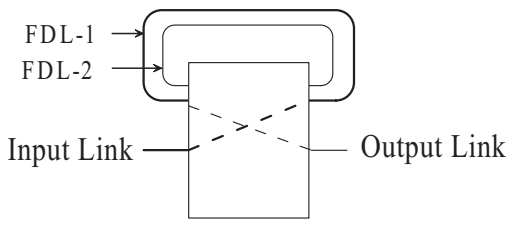

Fig. 1. An optical TSI example which consists of an optical space switch and FDLs.

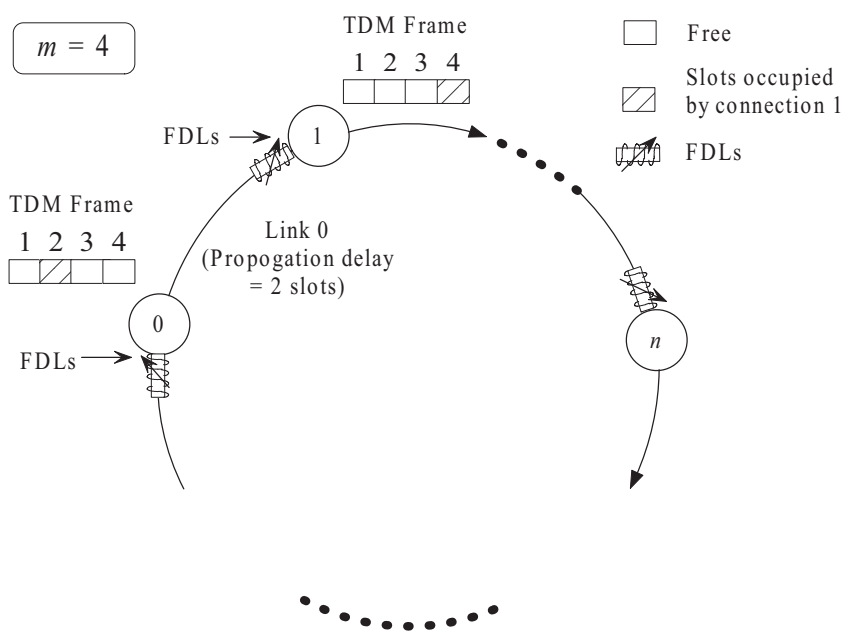

Fig. 2. A ring without TSI needs only simple fiber delay lines in each node to synchronize the incoming and outgoing slots. It is much easier and cheaper to build than a ring with TSI.

TSI-free OTDM ring, all slots form equal-length cycles and the slot cycle length will affect the efficiency of the ring.

Consider a unidirectional ring with $N$ nodes indexed from 0 to $(N-1)$ in the clockwise direction, such that data flows from node $i$ to node $((i+1) \bmod N)$ on the ring. Link from node $i$ to node $((i+1) \bmod N)$ is denoted as link $i$ (Fig. 2). The slot size is 1 and the frame size is $m$. Without loss of generality, we can assume that all nodes begin their frames at the same time and slot 0 is transmitted from all nodes simultaneously. Suppose the propagation delay on link 0 is 2 slots. Then the slot (node 0 , slot 1 ) will become (node 1 , slot 3 ) after the slot leaves node 1 (Fig. 2)

Time shifting occurs naturally as a result of propagation delay. This phenomenon is analogous to wavelength conversion in a WDM network. The only difference is that time slot shifting is done without any additional hardware. In contrast, wavelength shifting requires a wavelength converter.

If we trace a slot as it traverses the ring, we obtain a sequence of slot numbers. We discovered in [1] that any traced slot will eventually return to the original slot and form a cycle. We called this a slot cycle [1]. Furthermore, all slots in a TSI- 


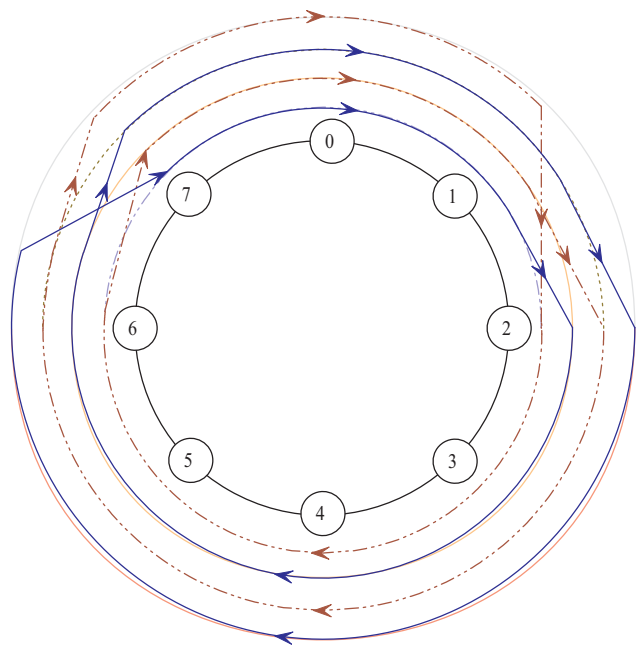

Fig. 3. There are 2 slot cycles assuming the propagation delays are 4 slots except link 1 and 6 (both have delay of 1 slot).

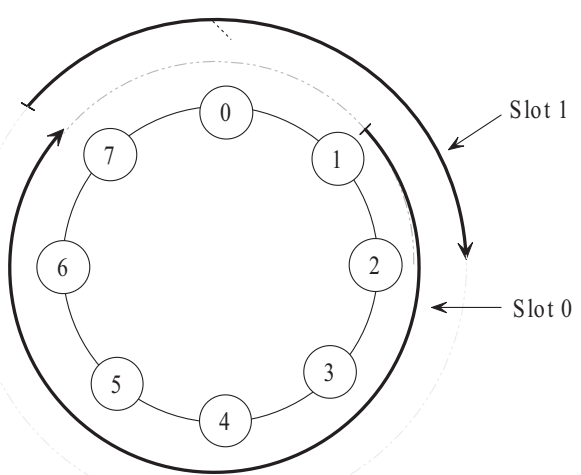

Fig. 4. One lightpath from node 1 to node 7 has been set up. If we intend to set up another lightpath from node 7 to node 2 , we have to use a different slot because the two paths overlap in the segment between node 1 and 2 .

free OTDM ring are divided into disjoint slot cycles and all slot cycles have the same length. One example is shown in Fig. 3 where $m=4, N=8$. The propagation delay equals 4 on all links except the links between nodes 1 and 2 and between nodes 6 and 7, which have propagation delay of 1 slot. There are two slot cycles in this example: one shown by the solid line and the other by the dashed line. Both cycles have the same length of 16 - each contains 16 time slots.

Another important result of [1] is that the length of the slot cycle has a strong bearing on the space re-use efficiency of each slot of the ring. An intuitive explanation of the problem is given in Fig. 4. To simplify the discussion, we assume that the propagation delay between two nodes is zero (or a multiple of the frame size) such that slot 0 sent out by node $i$ will be sent out again as slot 0 by node $(i+1)$. Suppose slot 0 from node 1 to node 7 has already been occupied by an existing lightpath, and now a new lightpath from node 7 to node 2 needs to be set up. Because the two lightpaths overlap in the segment between node 1 and node 2, we must use a different slot for the new connection. In other words, the remaining segment of slot 0 between node 7 and node 1 cannot be used for the second lightpath even when it is available.
This simple example is based on a unidirectional ring. Often rings are bidirectional and the space efficiency problem needs to be combined with the routing and slot assignment (RTA) problem. Routing refers to the selection of which directional ring to use, and slot assignment refers to which slot in a frame to choose. In this paper we will derive a general bound to characterize the ring efficiency and its relationship with the cycle length in a TSI-free bi-directional OTDM ring. We also discuss the RTA scheme that leads to the performance bound.

This work, as shown later, uses some results derived for WDM rings. If propagation delay $=0$, the RTA problem is equivalent to the RWA (routing and wavelength assignment) problem in a WDM network without wavelength converters [7][8]. But once delays are introduced, slot cycles are formed, and the RTA problem will have no counterpart in the WDM network.

\section{Space Reuse Efficiency and Cycle Length}

We consider a bidirectional OTDM ring network that consists of a clockwise ring and a counter-clockwise ring. To study the impact of the cycle length on the space use efficiency of a ring, one can use a general traffic matrix but then usually a simulation-based study is required. One may also select some special classes of traffic matrixes that allow easier analyses and closed-form solutions [7][8]. We use the latter approach in this paper. For an OTDM or WDM ring, the element $t_{i, j}$ in the traffic matrix represents the number of lightpaths to be set up from node $i$ to node $j$. Thus, a traffic matrix represents a virtual topology to be constructed on top of the ring. We focus on what is known as the single-port traffic matrix class. In a matrix of this class, each node can originate and terminate at most one lightpath. So the corresponding virtual topology will be a single loop or multiple loops. For example, assume the ring has 8 nodes. The topology represented by the following single-port traffic matrix

$\begin{array}{lllllllll}\text { From } & 0 & 1 & 2 & 3 & 4 & 5 & 6 & 7 \\ \text { To } & 7 & 0 & 5 & 2 & 3 & 1 & 4 & 6\end{array}$

forms one logical loop, and the topology of the following traffic matrix

$\begin{array}{lllllllll}\text { From } & 0 & 1 & 2 & 3 & 4 & 5 & 6 & 7 \\ \text { To } & 7 & 0 & 5 & 4 & 2 & 3 & 1 & 6\end{array}$

corresponds to two loops. In the following, we consider singleport traffic matrixes that correspond to a single-loop virtual topology, as done in [7][8]. Let a connection be denoted as a source-destination pair $\left(n_{k}, n_{j}\right)$. The two connections $\left(n_{k}, n_{j}\right)$ and $\left(n_{j}, n_{h}\right)$ are said to be adjacent. In a single-loop virtual topology, all $N$ lightpaths can be labeled into $N$ consecutive adjacent lightpaths. Our intention is to show the relationship between the space reuse efficiency of the ring and the slot cycle length.

We denote the propagation delay on link $i$ by $d_{i}$, where $d_{i}$ is an integer (i.e. a multiple of time slots). Denote $D$ as the total propagation delay of the ring; i.e. $D=d_{0}+d_{1}+\ldots+d_{N-1}$. In [1], we showed that the cycle length $L=x N$,where $x$ is the smallest integer that satisfies

$$
x D \bmod m=0 .
$$

Given $N$ and $x$, we will establish the theorem that $x\left\lceil\frac{N}{4 x-1}\right\rceil$ slots are always sufficient for the set up of a single- 
loop virtual topology. The theorem relies on the following lemma which outlines an efficient routing and wavelength assignment strategy.

Lemma 1: Suppose the slot-cycle length equals $x N$, we can always establish $(4 x-1)$ adjacent lightpaths in a pair of slotcycles, one from the clockwise ring and the other from the counterclockwise ring.

Proof: Denote these $(4 x-1)$ adjacent connections by 1 to $(4 x-1)$. Suppose that there is an artificial connection $4 x$ that connects the destination node of connection $(4 x-1)$ and the source node of connection 1 . Thus these $4 x$ connections form a one-loop logical topology. We use $L_{i}$ to denote the number of hops required to set up the connection $i$ in the clockwise direction. Let

$$
\sum_{i=1}^{4 x} L_{i}=v N
$$

where $v$ must be an integer as a single-loop virtual topology must form an integer number of cycles around the ring in the clockwise direction.

Let $\bar{L}$ denote the average number of hops required in the clockwise direction for these $4 x$ connections. We have

$$
\bar{L}=\frac{\sum_{i=1}^{4 x} L_{i}}{4 x} .
$$

Let $h=\lfloor x N / \bar{L}\rfloor$. It is shown in [8] that it is always possible to find a set of $h$ adjacent lightpaths with an average hop count $\tilde{L}$ less than or equal to $\bar{L}$. We call this set of $h$ adjacent lightpaths the clockwise set, and call the set of remaining ( $4 x-$ $h$ ) lightpaths the counterclockwise set. The total number of consecutive hops required to accommodate the clockwise set, denoted by $D_{c}$, in the clockwise direction, is

$$
D_{c}=h \tilde{L} \leq h \bar{L}=\lfloor x N / \bar{L}\rfloor \cdot \bar{L} \leq x N
$$

Thus we can put the clockwise set in the clockwise slot-cycle with size $x N$.

Next, consider the remaining $(4 x-h)$ lightpaths. Denote the average clockwise hop count of the counter-clockwise set by $\hat{L}$. Because $\tilde{L} \leq \bar{L}$, obviously $\hat{L} \geq \bar{L}$. If we route them through the counterclockwise ring, the average hop count must be $(N-\hat{L})$. Let $D_{c c}$ denote the total number of consecutive hops required to accommodate the counterclockwise set in the counterclockwise direction. Then we have

$$
\begin{aligned}
D_{c c} & =(4 x-h)(N-\hat{L}) \\
& \leq(4 x-h)(N-\bar{L})=\left(4 x-\left\lfloor\frac{x N}{L}\right\rfloor\right)(N-\bar{L})
\end{aligned}
$$

From Eq. (2) and (3), we get

$$
\bar{L}=(v N) /(4 x)
$$

Then we have

$$
D_{c c} \leq\left(4 x-\left\lfloor\frac{x N}{\bar{L}}\right\rfloor\right)(N-\bar{L})=\frac{N}{4 x}\left(4 x-\left\lfloor\frac{4 x^{2}}{v}\right\rfloor\right)(4 x-v)
$$

It can be shown that for integer $x$ and $v$, the last quantity in Eq. (7) is maximized when $v=2 x$, which leads to

$$
D_{c c} \leq \frac{N}{4 x}\left(4 x-\left\lfloor\frac{4 x^{2}}{v}\right\rfloor\right)(4 x-v) \leq x N
$$

Thus a counterclockwise slot-cycle with size $x N$ is sufficient to set up the counter-clockwise set.
We now use the preceding lemma to present our theorem which specifies the maximum number of slots needed to set up a single-loop virtual topology.

Theorem 1: In a bidirectional TSI-free OTDM ring, any single-loop logical topology can be implemented with at most $x\lceil N /(4 x-1)\rceil$ time slots in each direction, where $x$ is defined in Eq. (2).

Proof: A single-loop virtual topology consists of $N$ consecutive adjacent lightpaths (connections) in the worst case. Divide the lightpaths into sets of $(4 x-1)$ adjacent lightpaths. There will be $\lceil N /(4 x-1)\rceil$ sets and the last set may contain less than $(4 x-1)$ adjacent lightpaths. By Lemma 1, we can route each of these sets in a pair of slot-cycles of length $x N$. A slotcycle of length $x N$ traverses each link $x$ times and therefore consumes $x$ time slots. Thus, any single-loop connection pattern can be implemented with at most $x\lceil N /(4 x-1)\rceil$ slots in each direction.

Theorem 1 provides a bound on the capacity (the number of slots) needed for setting up a single-loop virtual topology. This bound is itself lower-bounded by $\lceil N / 4\rceil$. Theorem 1 , by considering an arbitrary cycle length $x N$, supplements our result in [1], where we assumed that all the slots in the ring is connected in one slot cycle and proved that a capacity of $\lceil N / 4\rceil$ slots is always sufficient to accommodate any single-loop virtual topology with $N$ lightpaths. Theorem 1 is applicable when the cycle length $x N$ is insufficient to accommodate all the lightpaths.

\section{CONCLUSION}

Time shifting is the main difference between an OTDM ring and a WDM ring. With time-slot corresponding to wavelength, time shifting in OTDM is analogous to forced wavelength conversion in WDM. While time shifting occurs naturally as a result of propagation delay, wavelength conversion in the WDM is not possible without wavelength converters. In this paper we have derived a general bound to characterize the ring efficiency and showed its relationship with the cycle length in a TSI-free bidirectional OTDM ring. We also discussed the RTA scheme that leads to the performance bound.

\section{REFERENCES}

[1] A. Chen, C.-T. Lea, and A. Wong," "A new optical ring architecture," IEEE Trans. Commun., vol. 55, pp. 2134-2141, Nov. 2007.

[2] N.-F. Huang, G.-H. Liaw, and C.-P. Wang, "A novel all optical transport network with time-shared wavelength channels," IEEE J. Select. Areas Commun., vol. 18, pp. 1863-1875, Oct. 2000.

[3] B. Wen and K. M. Sivalingam, "Routing, wavelength and time-slot assignment in time division multiplexed wavelength-routed optical wavelength networks," in Proc. IEEE INFOCOM 2002.

[4] A. Chen, A. Wong, and C. T. Lea, "Routing and time-slot assignment in optical TDM networks," IEEE J. Select. Areas Commun., vol. 22, Nov. 2004.

[5] B. Li, Y. Qin, X.-R. Cao, and K. M. Sivalingam, "Photonic packet switchings: architecture and performance," SPIE Optical Networks, vol. 2, pp. 27-39, Jan./Feb. 2001.

[6] S. Yao, B. Mukherjee, and S. Dixit, "Advances in photonic packet switching: An overview," IEEE Commun. Mag., vol. 38, pp. 84-94, Feb. 2000.

[7] A. Narula-Tam, P. J. Lin, and E. Modiano, "Efficient routing and wavelength assignment for reconfigurable wdm networks," IEEE J. Select. Areas Commun., vol. 20, no. 1, pp. 75-88, Jan. 2002.

[8] L.-W. Chen and E. Modiano, "Efficient routing and wavelength assignment for reconfigurable wdm networks with wavelength converters," IEEE/ACM Trans. Networking, vol. 13, no. 1, pp. 173-186, Feb. 2005. 